\title{
Lightning Surges on a Control Cable Incoming through a Grounding Lead
}

\author{
Akihiro Ametani Member (Doshisha University) \\ Norikazu Taki Student Member (Doshisha University) \\ Daisuke Miyazaki Student Member (Doshisha University) \\ Naoto Nagaoka Member (Doshisha University) \\ Shigemitu Okabe Member (Tokyo Electric Power Company)
}

Keywords: lightning surge, low-voltage control cable, grounding mesh, sheath grounding, induced voltage

The present paper investigates induced voltages to a control cable based on EMTP simulations. A simulation neglectiong mutual coupling between a control cable and a grounding mesh gives voltages on the cable core produced by a circulating current between the cable sheath and the mesh due to sheath grounding. An induced voltage from the lightning current along the grounding mesh is also investigated considering the current flowing into the soil. The effect of cable sheath grounding and a grounding lead is also discussed.

Figure 1 illustrates a model circuit for investigating transient voltages and currents on a control cable.

Figure 2 shows simulation results with mutual coupling between a control cable and a counterpoise. It is clear in Fig. 2(a) that only an induced voltage, about $2.5 \mathrm{~V}$, due to a counterpoise current appears on the core and the sheath, because the sheath is not grounded. It is observed that the current on the sheath is nearly the same as that with no mutual coupling, and the cable voltages at the both ends are given as the sum of those with no mutual coupling and the induced voltages in Fig. 2(a). (a) and (b) shows no diffrence of a core voltage and thus, the core voltage is an induced voltage from the counterpoise. Figure 2 (c) shows a twice greter core voltage compared with (a) and (b), and thus a half the core voltage in (c) is a result of a sheath circulating current because of sheath both ends grounding. No significant difference is observed for the counterpoise voltages and currents with and without mutual coupling as is celar from (b) and (c) in comparison with (a).

Based on investigations of lightning surges on a control cable incoming through a grounding mesh, the following remarks have been obtained.

(1) So-called induced voltages on a control cable is a sum of an induced voltage due to a current on a counterpoise and of a voltage transfered from the counterpoise. When the both ends of the cable are grounded, a circulating current in a closed loop composed of the

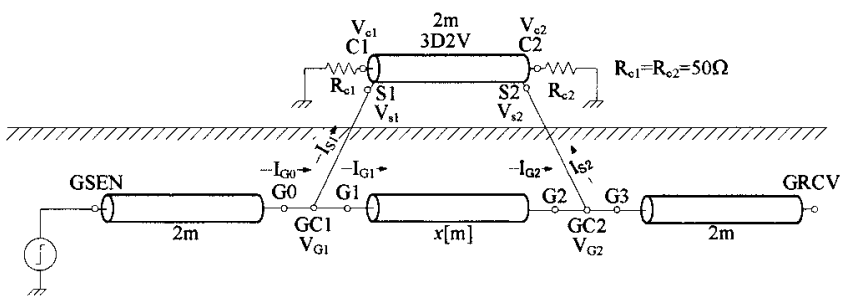

Fig. 1. A model circuit cable sheath and the counterpoise affects significantly the core voltage at the receiving end which becomes the largest among various sheath grounding.

(2) When the parallel length of the control cable and the counterpoise is long, current decrease along the counterpoise should be taken into account to evaluate an induced voltage to the cable core due to mutual coupling between the cable and the counterpoise.

(3) The inductance of a grounding lead reduces a current flowing into the sheath because of its high impedance for a high frequency transient. At the same time, a current flowing into the counterpoise parallel to the cable increases and the current results in a higher induced voltage to the cable core due to mutual coupling between the cable and the counterpoise.

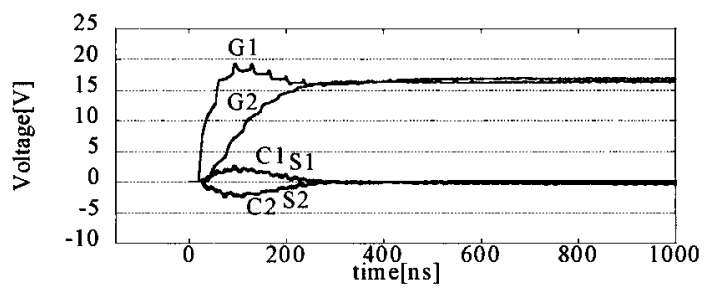

(a) Sheath not grounded to counterpoise

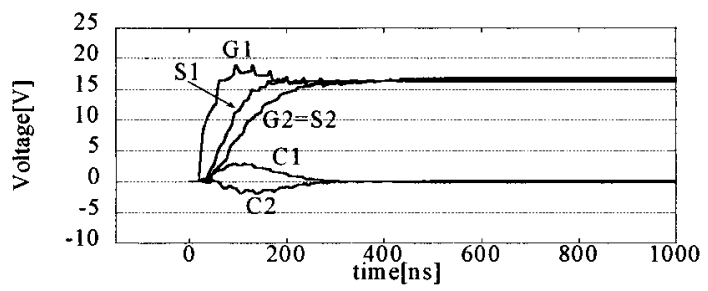

(b) Receiving-end sheath grounded to counterpoise

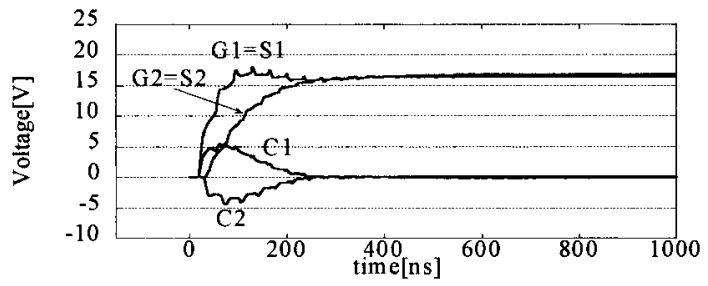

(c) Sheath both-end grounded to counterpoise

Fig. 2. Simulation results of voltages considering mutual coupling $(x=2 \mathrm{~m})$ 


\title{
Lightning Surges on a Control Cable Incoming through a Grounding Lead
}

\author{
Akihiro Ametani* Member \\ Norikazu Taki* Student Member \\ Daisuke Miyazaki* Student Member \\ Naoto Nagaoka* Member \\ Shigemitu Okabe** Member
}

The present paper has investigated the effect of sheath grounding on a control cable based on EMTP simulations, when a lightning current flows into counterpoises representing a part of a grounding mesh. Voltages and currents observed on the control cable are given as a superposition of an well-known induced voltage due to the lightning current on the counterpoise, of a voltage transferred from the counterpoise to which the cable metalic sheath is grounded, and of a lightning current circulating in a closed loop composed of the metalic sheath and the counterpoise when the cable both ends are grounded. The induced voltage from the counterpoise is not necessarily dominant even in a long cable because the current along the counterpoise decreases rapidly as the distance from the sending end increases. Although the sheath grounding decreases the node voltage due to the induced voltage, but at the same time increases a voltage transferred from the counterpoise. The inductance of a grounding lead reduces a current flowing into the cable sheath during a high frequency transient, and thus a more current flows through the counterpoise. This results in a higher induced voltage to the control cable from the counterpoise.

Keywords: lightning surge, low-voltage control cable, grounding mesh, sheath grounding, induced voltage

\section{Introduction}

Electromagnetic interference becomes more and more significant in control circuits of generator stations and substations as the number of digital control equipments increases. A survey of failures and mulfunctions of low voltage control equipments in generator stations and substations shows that nearly 70 percents of the failures are caused by lightning surges ${ }^{(1)(2)}$. The lightning current flows into a grounding mesh in a station and it has been understood that the current causes an induced transient voltage on a control cable. Because the metalic sheath of the control cable is grounded to the mesh as recommended by a standard and a guideline for the electromagnetic interference ${ }^{(2)-(5)}$, the cable sheath voltage becomes the same as that of the grounding mesh. If the both ends of the metalic sheath are grounded following the IEC/CIGRE recommendation, a current is circulating in a closed loop composed of the cable sheath and the grounding mesh. The circulating current induces a voltage to the cable core, and the core voltage is given as a vector sum of the induced voltages from the lightning current along the grounding mesh and the circulating current. Also, the lightning current along conductors of the mesh flows into the soil and becomes a function of the length of the conductor ${ }^{(6)}$. Thus, the induced voltage to the cable is a function of the conductor length.

\footnotetext{
* Faculty of Engineering, Doshisha University

Tatara Miyakodani, Kyo-Tanabe 610-0321

** Tokyo Electric Power Company

4-1, Egasaki-cho, Tsurumi-ku, Yokohama 230-8510
}

The above fact seems not well understood, and a detailed analysis of the components, i.e. (1) the induced voltage due to mutual coupling from the lightning current along the grounding mesh, (2) the counterpoise voltage transferred to the cable sheath because of the sheath grounding, and (3) the circulating current due to sheath both-end grounding, has not been made. The reason for this is that all the measured results have involved the above components ${ }^{(2)(7)-(9)}$.

The present paper investigates induced voltages to a control cable based on EMTP simulations. It is easy to evaluate the above mentioned components separately in the EMTP simulations. That is, a simulation neglecting mutual coupling between a control cable and a grounding mesh gives voltages on the cable by grounding the cable sheath to the mesh. An induced voltage from the lightning current along the grounding mesh is also investigated considering the current flowing into the soil. The effect of cable sheath grounding and a grounding lead is also discussed.

\section{Model Circuit}

Figure 1 illustrates a model circuit for investigating transient voltages and currents on a control cable. A counterpoise representing a part of a grounding mesh is buried at the depth of $h_{g}=0.3 \mathrm{~m}$ from the earth surface. The counterpoise is a cupper cylinder with the outer radius of $r_{g}=2.5 \mathrm{~cm}$, and the earth resistivity is $100 \Omega \mathrm{m}$. As a control cable, a 3D2 V cable, of which the cross-section and the physical parameters are given in Fig. 2, is suspended at the height of $h_{c}=0.1 \mathrm{~m}$ above the earth surface. A step-function current with the amplitude 


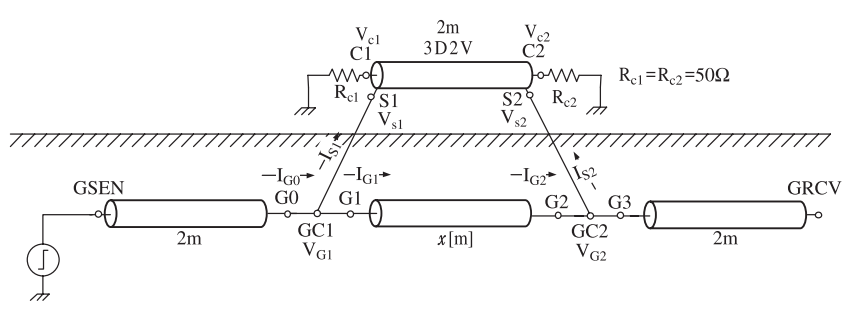

Fig. 1. A model circuit

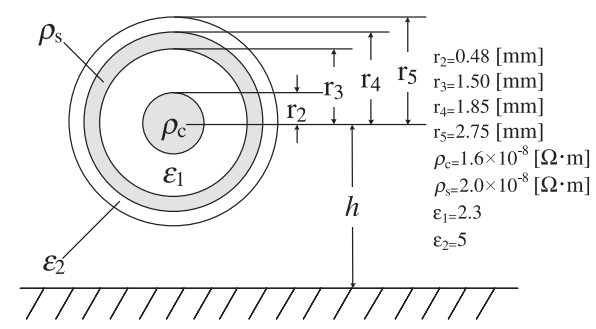

Fig. 2. A control cable (3D2V)

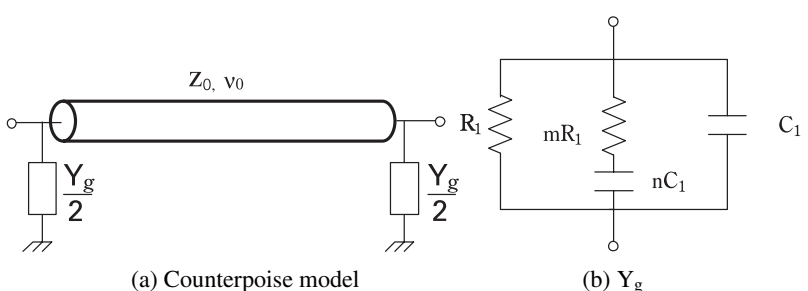

Fig. 3. A model circuit of a counterpoise

of $1 \mathrm{~A}$ is applied to the sending end of the counterpoise as in Fig. 1. Transient voltages and currents on the control cable and the counterpoise are calculated by EMTP.

A counterpoise is represented by a model circuit illustrated in Fig. 3 which is composed of a distributed line with the surge impedance $Z_{0}$ and the propagation velocity $v_{0}$ and a shunt admittance $\mathrm{Y}_{\mathrm{g}}{ }^{(10)}$. In this paper, $\mathrm{m}$ and $\mathrm{n}$ are set to be 1 and 5 respectively. Fig. 4 shows a comparison of experimental results and EMTP simulation results by the model circuit of Fig. 3 for transient voltages and currents along the counterpoise with the total length of $6 \mathrm{~m}$ in Fig. 1. The figure shows that the accuracy of the counterpoise model in Fig. 3 is satisfactory in comparison with the measured results.

In a simulation of an induced voltage to an overhead control cable from a counterpoise, the control cable and the counterpoise are represented as a distributed parameter line in the EMTP [11]. The parameters of the line models are evaluated by the EMTP Cable Parameters (CP) [12]. First, the model system is evalated as an overhead line system by the CP with a negative sign of the depth of the counterpoise. By the input date, the $\mathrm{CP}$ gives self impedance/admittance of the overhead cable and the mutual impedance to the counterpoise. Then the self impedance/admittance of the counterpoise is calculated as an underground cable. Finally, the self impedance/admittance of the counterpoise in the first calculation is replaced by those in the second one.

Table 1 gives simulation conditions such as grounding of the metalic sheath of a control cable, and results of maximum voltages and currents. In the table, case $X i-L j$ represents:

$X=\mathrm{A}: x=2[\mathrm{~m}], \mathrm{B}: x=10[\mathrm{~m}], \mathrm{C}: x=20[\mathrm{~m}]$

$\mathrm{D}: x=2[\mathrm{~m}]$ and two more counterpoises connected to

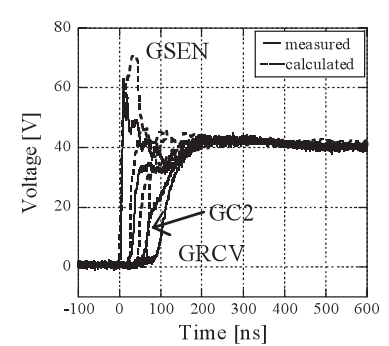

(a) Voltage

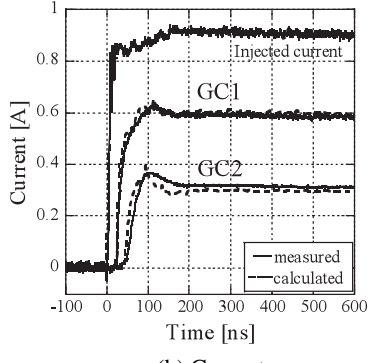

(b) Current
Fig. 4. Comparison of measured and simulation results of counterpoise voltages and currents

Table 1. Simulation conditions and results

(a) No mutual coupling (Case $X 0-L j$ )

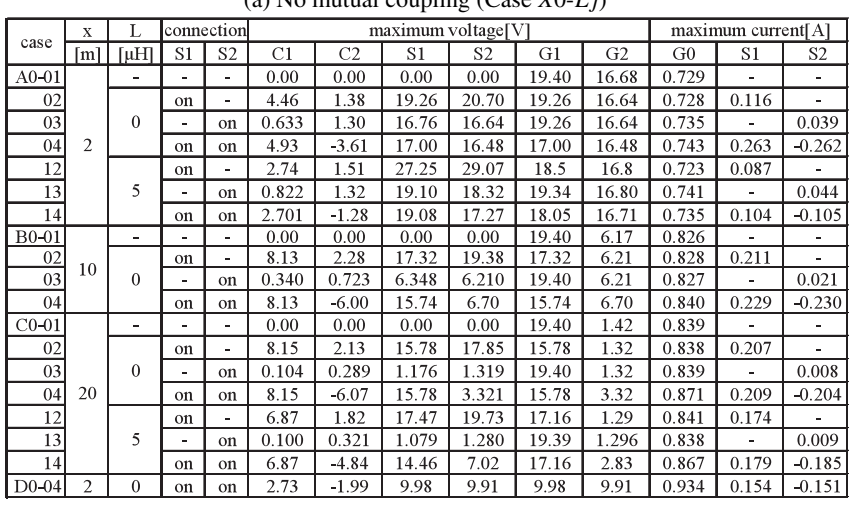

(b) With mutual coupling (Case $X 1-L j$ )

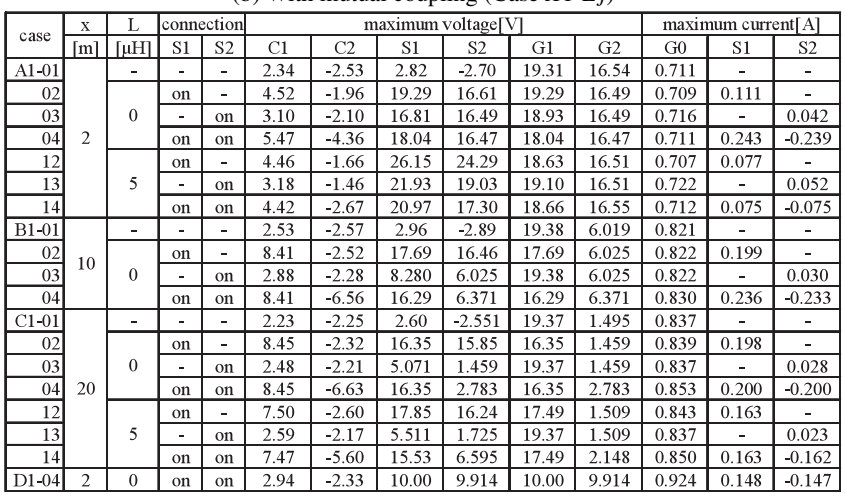

node GC1

$i=0$ : no mutual coupling between control cable and counterpoise

$i=1$ : mutual coupling between control cable and counterpoise

$L=0$ : no inductance considered for a grounding lead

$L=5: 5 \mu \mathrm{H}$ for a lead wire

$j$ for grounding of metalic sheath to counterpoise

$j=1$ : no grounding, $j=2$ : sending end grounded

$j=3$ : receiving end, $j=4$ : both ends

\section{Simulation Results and Discussions}

Figures 5 to 12 show simulation results of transient voltages and currents. The peak values are summarized in Table 1 .

3.1 No Mutual Coupling (Case $X 0-L j$ )

3.1.1 Case A0-0 $j: x=2 \mathrm{~m}, \boldsymbol{L}=\mathbf{0} \quad$ Figure 5 is the case of neglecting mutual coupling between a control cable and a counterpoise with the parallel length $x=2 \mathrm{~m}$. Thus, 

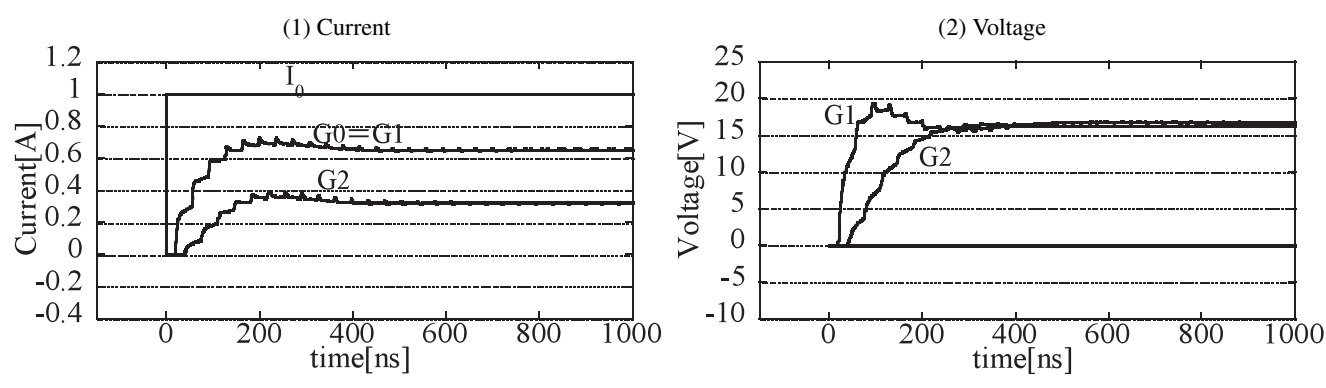

(a) Sheath not grounded to counterpoise (CaseA0-01)
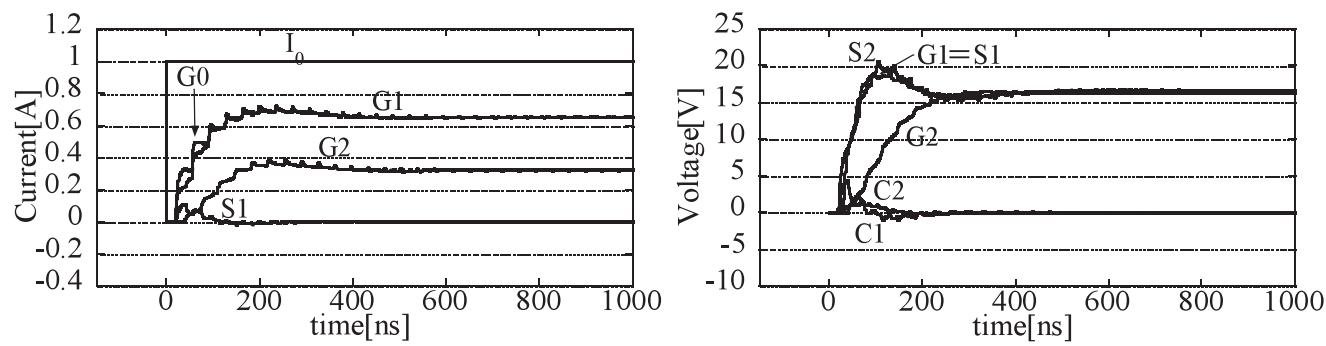

(b) Sending-end sheath grounded to counterpoise (CaseA0-02)
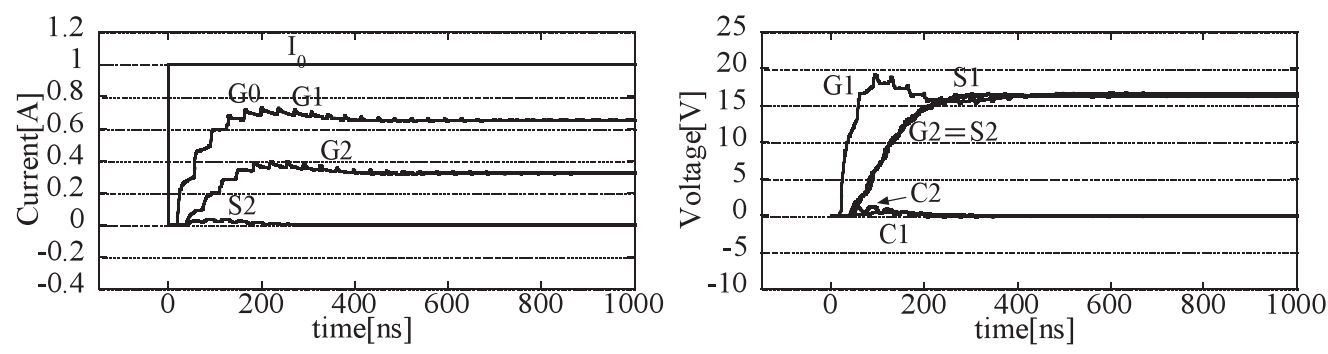

(c) Receiving-end sheath grounded to counterpoise (CaseA0-03)
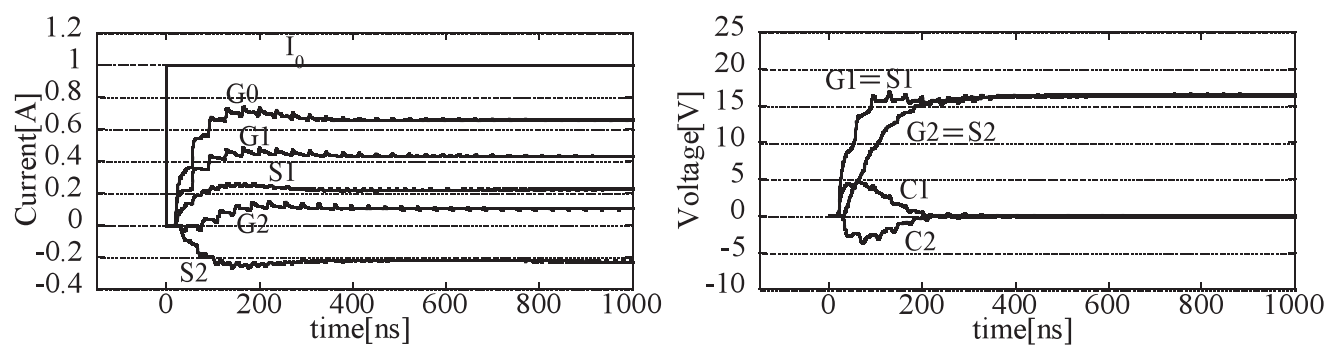

(d) Sheath both-end grounded to counterpoise (CaseA0-04)

Fig. 5. Simulation results neglecting mutual coupling between the control cable and the counterpoise $(x=2 \mathrm{~m})$

the voltages and currents on the control cable in the figure are caused only by connecting (grounding) the metalic sheath of the cable to the counterpoise.

No voltage and no current appear on the control cable in Fig. 5(a) for there is no connection nor mutual coupling between the cable and the counterpoise. When one end of the sheath is grounded to the counterpoise, a voltage and a current appear on the grounded node. In case A0-02 (sendingend sheath to counterpoise), the sheath voltage at the sending end S1 becomes nearly the same as the counterpoise voltage of about $20 \mathrm{~V}$ at the grounded node G1, and the core voltage at the sending end $\mathrm{C} 1$ reaches $4 \mathrm{~V}$ as observed in Fig. 5(b)-(2). Also, a small voltage of about $1 \mathrm{~V}$ is observed at the core receiving-end. The core voltages are produced by electro-static and magnetic coupling between the cable core and the sheath during an initial transient period up to about $300 \mathrm{~ns}$ as a small current, about $0.1 \mathrm{~A}$, on the cable sheath at the sending end is observed in Fig. 5(b)-(1) during the time period. A similar trend is observed for the receivingend sheath grounded to the counterpoise (Case A0-03), that is, the sheath voltage becomes nearly the same as the counterpoise voltage at the receiving end. Small voltages appear on the both ends of the core and a very small current is observed on the sheath in Fig. 5(c). It should be noted that the core voltages and the sheath current are much smaller than those in Case A0-02.

When the both ends of the cable sheath are grounded to the counterpoise, a large current, nearly $0.25 \mathrm{~A}$ which is about $30 \%$ of the current $\mathrm{I}_{\mathrm{G} 0}$ flowing into node $\mathrm{GC} 1$ of the counterpoise, flows into the sheath as in Fig. 5(d)-(1), and results in nearly $5 \mathrm{~V}$ at the core sending end and $-3.5 \mathrm{~V}$ at the receiving end as observed in Fig. 5(d)-(2). The core voltages are generated by electromagnetic coupling between the sheath and the core. It should be noted that the counterpoise voltages and currents are not much affected by the sheath grounding.

In summary, grounding the metalic sheath of a control 

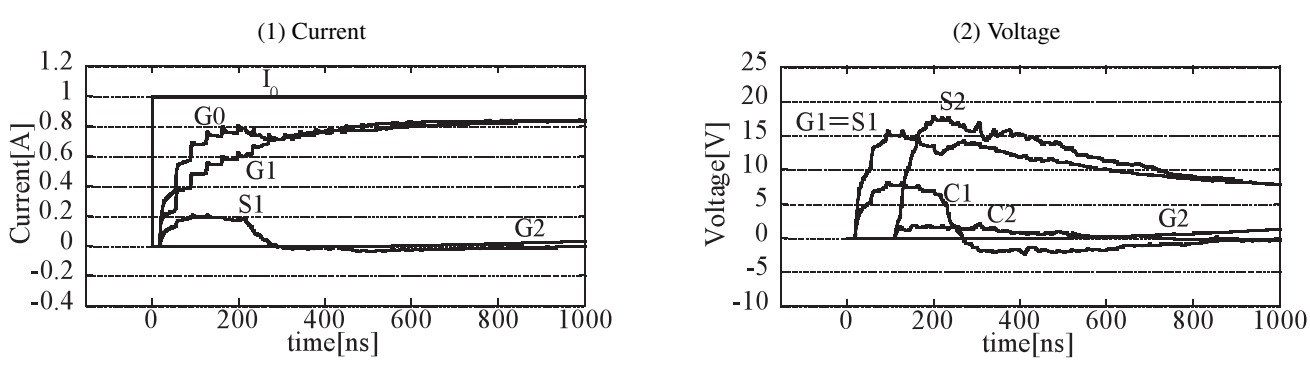

(a) Sending-end sheath grounded to counterpoise (CaseC0-02)
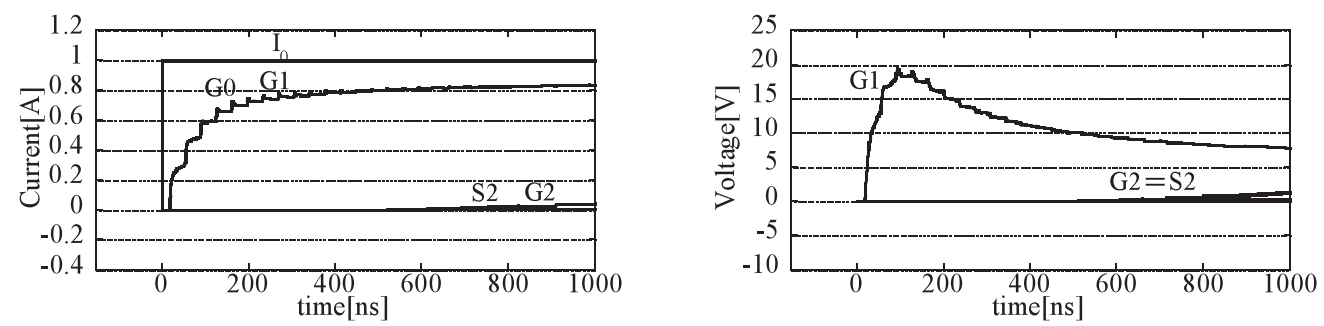

(b) Receiving-end sheath grounded to counterpoise (CaseC0-03)
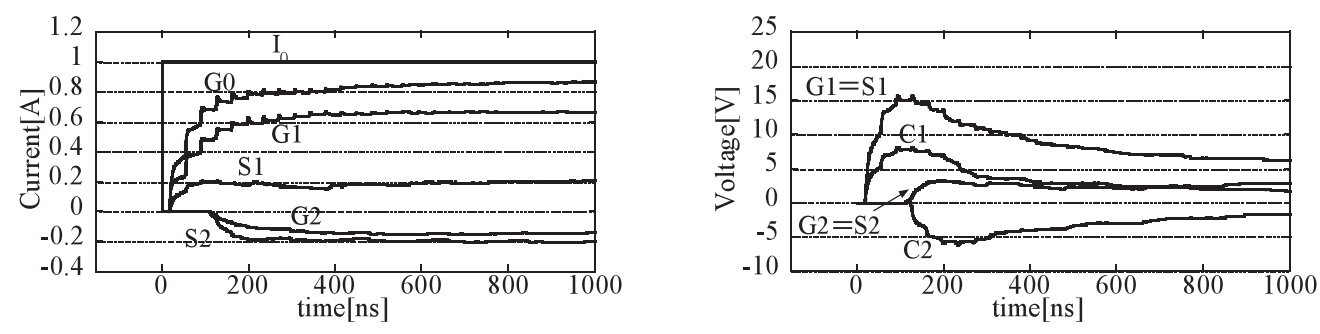

(c) Sheath both-end grounded to counterpoise (CaseC0-04)

Fig. 6. Effect of parallel length $x$ with no mutual coupling

cable to a counterpoise results in a current flowing into the sheath and generating core voltages due to electromagnetic and static coupling between the cable core and the sheath. Especially the grounding of the sheath both ends to the counterpoise produces a sheath current of about $30 \%$ of the counterpoise current and the voltage propotional to the current appears on the core during the initial time period of a transient. Grounding the sending-end sheath to the counterpoise also produces a rather high voltage to the core at the sending end.

3.1.2 Effect of length $x \quad$ Figure 6 shows the case of no mutual coupling with the parallel length $x=20 \mathrm{~m}$ between a control cable and a counterpoise. It is clear that currents at the sending end of the counterpoise are greater and the voltages are smaller than those in Fig. 5 for $x=2 \mathrm{~m}$, because the longer counterpoise length decreases its impedance seen from the sending end. Also, the currents along the counterpoise show a smooth increasing characteristic while the voltage at the sending end takes the peak at around $t=250 \mathrm{~ns}$ and then converges to a certain value. The above explained characteristics of the counterpoise agree with those for measured results ${ }^{(6)-(9)}$.

The sheath currents in Fig. 6 for $x=20 \mathrm{~m}$ show a remarkable difference from those in Fig. 5 for $x=2 \mathrm{~m}$. The current in Fig. 6(a)-(1) for the sending-end sheath grounded to the counterpoise is nearly a twice of that in Fig. 5(b)-(1), and is sustained up to $160 \mathrm{~ns}$ corresponding a twice the propagation time of a traveling wave on the sheath from the sending end to the receiving end. Corresponding to this fact, the sendingend core voltage reaches $8 \mathrm{~V}$ which is about 2 times of that in Fig. 5(b). A similar trend is observed in Fig. 6(c) for $x=20 \mathrm{~m}$ in comparison with Fig. 5(d) for $x=2 \mathrm{~m}$. It is quite clear in Fig. 6(c)-(1) that there exists a cerculating current between the control cable sheath and the counterpoise, i.e. current $\mathrm{S} 1$ at the sheath sending end and current S2 at the receiving end. The sheath current produces the core voltages about $8 \mathrm{~V}$ at the sending end and $-6 \mathrm{~V}$ at the receiving end which are much greater than those in Fig. 5(d)-(2). On the contrary, the cable voltages and current in Fig. 6(b), the receiving-end sheath connected to the counterpoise, are much lower than those in Fig. 5(c), because the counterpoise current at the receiving end becomes much smaller due to current penetration into the earth. It should be noted that the voltage difference between the cable core and the counterpoise is large at the sending end as in Table 1(a), but that at the receiving end becomes much smaller in comparison with the case of $x=2 \mathrm{~m}$.

\subsection{With Mutual Coupling (Case $X 1-L j$ )}

3.2.1 Case $\mathrm{A1-0} \boldsymbol{j}: \boldsymbol{x}=\mathbf{2} \mathrm{m}, \boldsymbol{L}=\mathbf{0} \quad$ Figure 7 shows simulation results with mutual coupling between a control cable and a counterpoise corresponding to Fig. 5 with no mutual coupling. It is clear in Fig. 7(a) that only an induced voltage, about $2.5 \mathrm{~V}$, due to a counterpoise current appears on the core and the sheath, because the sheath is not grounded to the counterpoise. The polarity at the receiving end is opposite to that at the sending end as in Ref. (13). It is observed in Fig. 7(b) that the current on the sheath is nearly the same as that in Fig. 5(b) and the core voltages at the both ends are given as the sum of those in Fig. 5(b) and the induced voltages in Fig. 7(a). The same observation is made for Fig. 7(c) in comparison with Fig. 5(c). It should be noted that Fig. 7(d) is nearly the same as Fig. 5(d). This means that the 

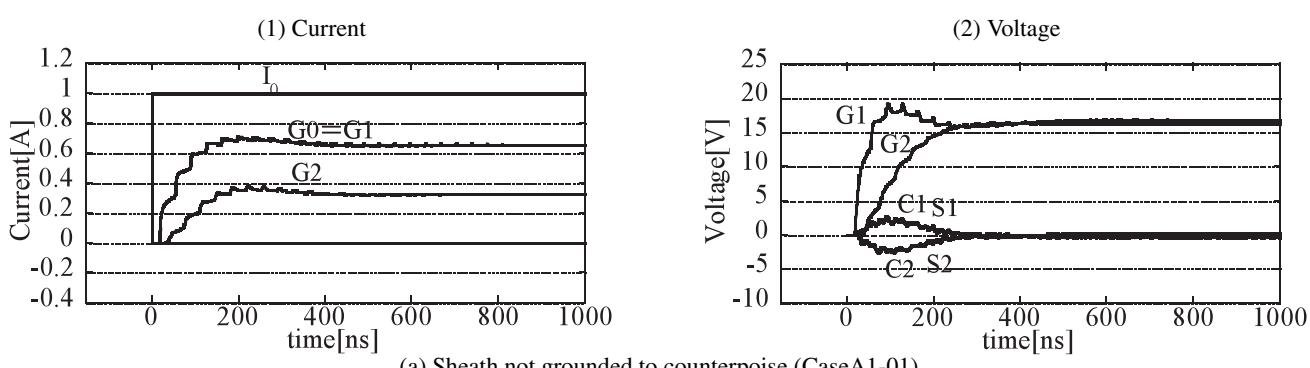

(a) Sheath not grounded to counterpoise (CaseA1-01)
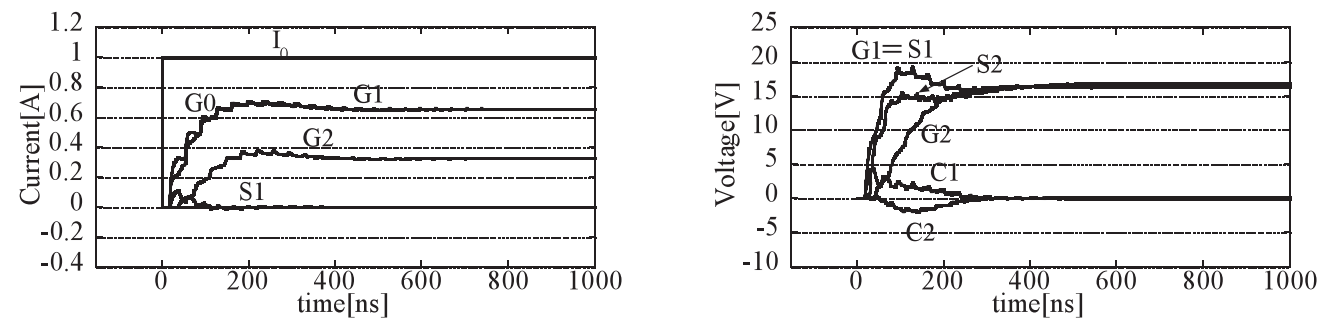

(b) Sending-end sheath grounded to counterpoise (CaseA1-02)
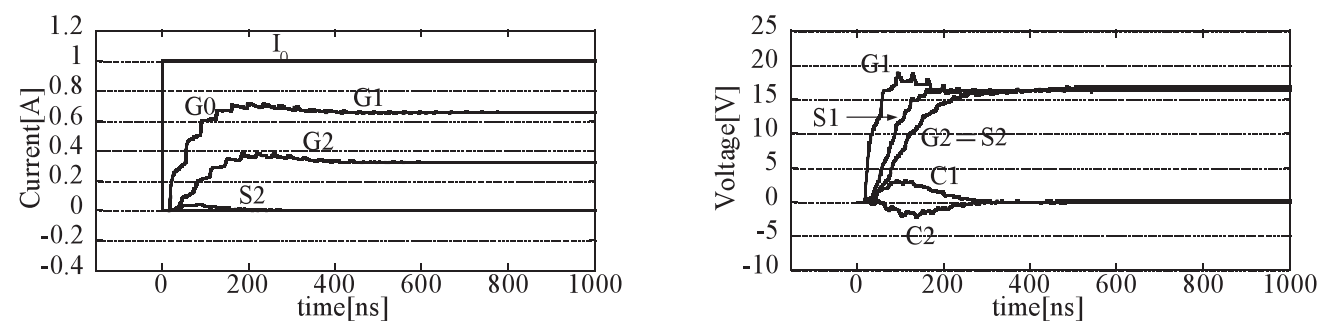

(c) Receiving-end sheath grounded to counterpoise (CaseA1-03)
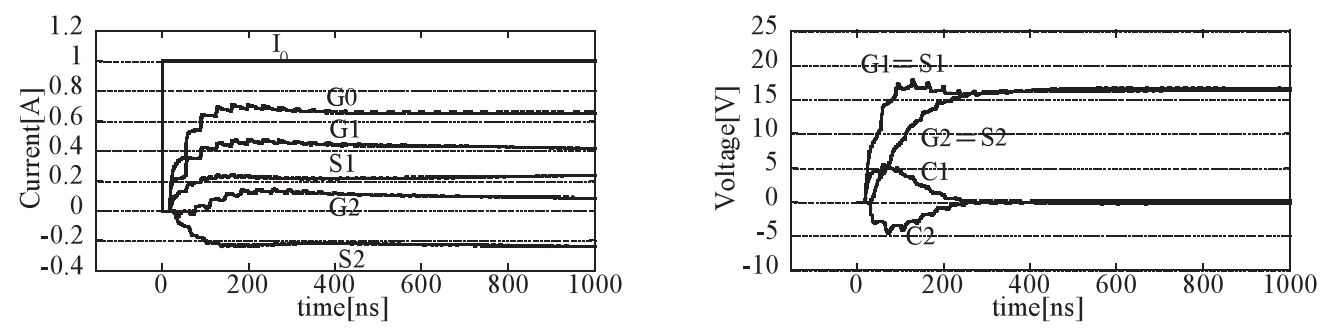

(d) Sheath both-end grounded to counterpoise (CaseA1-04)

Fig. 7. Simulation results considering mutual coupling between the control cable and the counterpoise $(x=2 \mathrm{~m})$

core voltage in Fig. 7(d) is generated by a circulating current between the sheath and the counterpoise, although the induced voltage, $2.5 \mathrm{~V}$, by the current on the counterpoise is decreased by the both-end grounding. If the sheath is grounded to another counterpoise on which no lightning current flows, the sheath both-end grounding results in the smallest voltage on the cable core as recommended by standards and guides ${ }^{(2)-(5)}$. The recommendation is correct only in the case of no lightning current on a counterpoise to which the sheath is grounded. No significant difference is observed for the counterpoise voltages and currents between Figs. 5 and 7.

In summary, the sending-end voltage is the largest in the sending-end and the both-end grounded cases. The receivingend voltage becomes the largest in the both-end grounded case because of a circulating current in a closed loop composed of the cable metalic sheath and the counterpoise. Thus, the receiving-end grounding is preferable than the sending and the both-end grounidng. The ovservations agree qualitatively with measured results in references ${ }^{(2)(7)-(9)}$, especially in Ref. (9) for the same 3D2 V cable.
3.2.2 Effect of length $x \quad$ Figure 8 shows simulation results of voltages for $x=20 \mathrm{~m}$. Current waveforms are not shown because those are nearly the same as Fig. 6-(1). It is clear in Fig. 8(a) for sheath not grounded to counterpoise that the induced voltages to the core and the sheath from the counterpoise are far greater than those in Fig. 7(a) for $x=2 \mathrm{~m}$. This is reasonable because an induced voltage is propotional to the parallel length of the inducing circuit, as is well-known ${ }^{(13)}$. The length for Fig. 8 being $20 \mathrm{~m}$, a 10 times greater induced voltage than that in Fig. 7 is expected if it is in a steady state, but the induced voltage at node $\mathrm{C} 1$ is about $2 \mathrm{~V}$ in Fig. 8(a) which is smaller than that in Fig. 7. The reason for the smaller induced voltage than that expected from the theory of steady-state induction is that the inducing current on the counterpoise decreases as the distance from the sending end increases as shown in Fig. 9. It is clear in Fig. 9 that the current along the counterpoise is decreasing exponentially as the distance increases. Furthermore, the rise time of the current, i.e. di/dt decreases rapidly. The transient induced voltage is given as: 


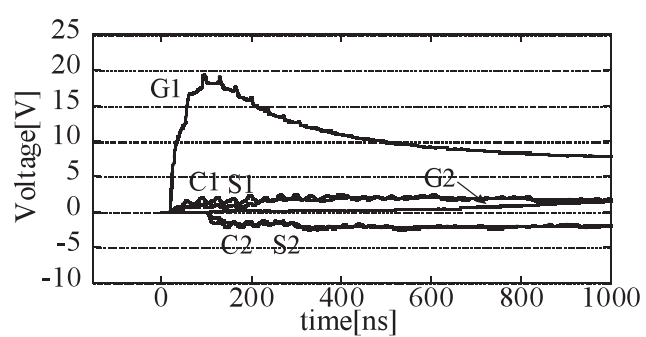

(a) Sheath not grounded to counterpoise (CaseC1-01)

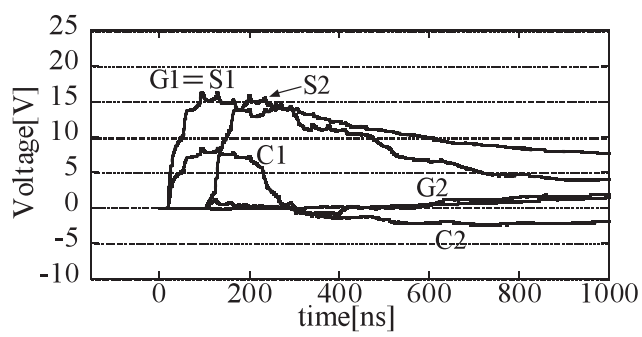

(b) Sending-end sheath grounded to counterpoise (Case C1-02)

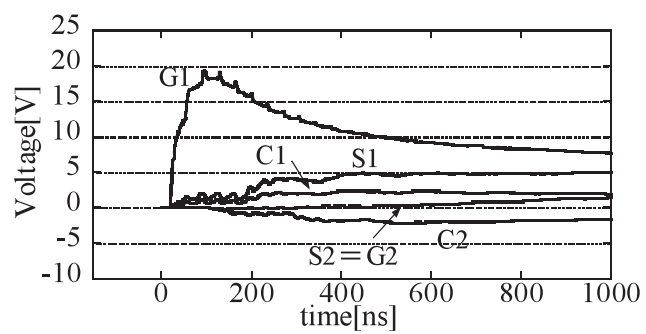

(c) Receiving-end sheath grounded to counterpoise (Case C1-03)

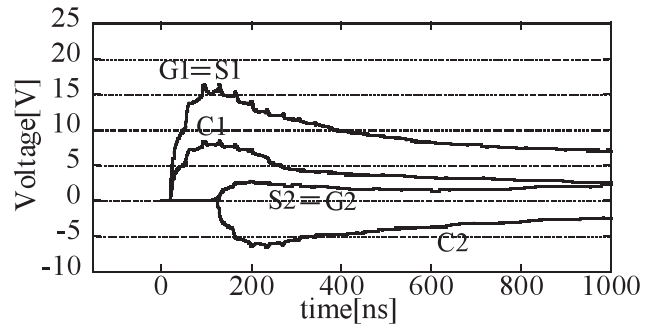

(d) Sheath both-end grounded to counterpoise (Case C1-04)

Fig. 8. Effect of parallel length

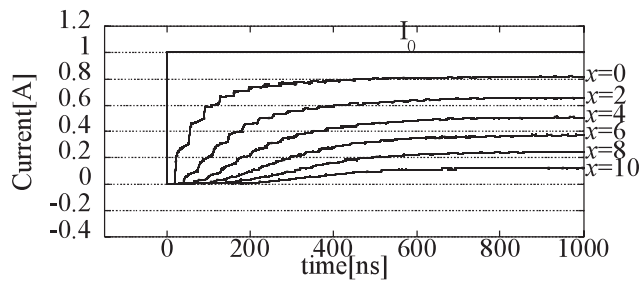

Fig. 9. Currents along the counterpoise at the distance $x$ from the sending end

$$
v_{m}(t)=M d i / d t \ldots \ldots \ldots \ldots \ldots \ldots \ldots \ldots \ldots \ldots
$$

where M: mutual inductance

It sholud be clear from the above equation that the transient induced voltage decreases rapidly in propotion to the rise time decrease of the inducing current.

The core voltages in Fig. 8(b) to (d) are given as the sum of the induced voltage in Fig. 8(a) and the voltage in Fig. 6 due to the sheath grounding to the counterpoise.Thus, the sending-end core voltage reaches nearly $8 \mathrm{~V}$ in Fig. 8(b) and (d), and the receiving-end core voltage is about $-7 \mathrm{~V}$ in

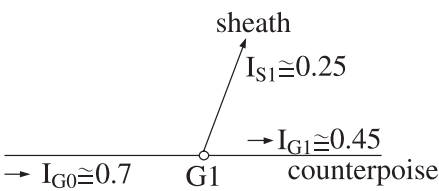

(a) A single counterpoise

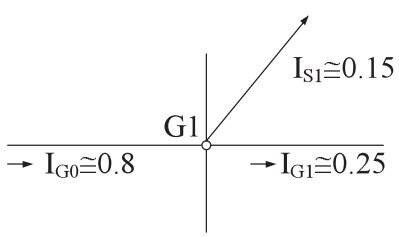

(b) Counterpoises connected at node G1

Fig. 10. Crossing counterpoises at node G1

Fig. 8(d), and -6V in Fig. 8(c).

3.2.3 Effect of crossing counterpoises (part of a mesh)

In practice, the metalic sheath of a control cable may be grounded to a node of a grounding mesh where mesh branches, i.e. counterpoises, are connected each other as illustrated in Fig. 10. The impedance at node G1 seen from the left in Fig. 10(b) becomes about $1 / 3$ of that in Fig. 10(a) on which all the previous investigations are based. The impedance variation in Fig. 10(b) results in current distribution different from that in Fig. 10(a), and thus, a current flowing into the cable sheath connected to node G1 differs. An example is presented in the figure. It is observed in Fig. 10 that the current flowing into the sheath is about $30 \%$ of the original current $\mathrm{I}_{\mathrm{G} 0}$ on the counterpoise when the sheath is grounded to an intermediate node of a single counterpoise as in (a), while it is less than $20 \%$ when the sheath is grounded to the mesh node as in (b). This difference certainly results in different transient voltages and currents on a control cable as shown in Table 1.

A comparison of case D1-04 with caseA1-04 and D0-04 with A0-04 in Table 1 makes clear that the cable voltages are reduced to less than a half by grounding the sheath to the node of a grounding mesh where counterpoises are connected to each other. The result is reasonable because of smaller current flowing into the sheath $\left(\mathrm{I}_{\mathrm{S} 1}\right)$ and that on the counterpoise to the right of node $\mathrm{G} 1\left(\mathrm{I}_{\mathrm{G} 1}\right)$ which is the inducing current to produce the cable voltage when the sheath is not grounded to the counterpoise.

The above result has indicated that an analysis of the induced voltage to the control cable can be carried out assuming a single counterpoise as the serverest case. Also, it should be mentioned that counterpoises which are a part of grounding mesh parallel to a control cable induces voltages on the cable. The voltage induced by the counterpoise nearest to the cable is the largest, and those due to the other counterpoises are much smaller because of the distance to the cable, and are somehow cancelled out because of the symmetrical configuration of the counterpoises to the cable. Therefore, it is expected that only the induced voltage from the nearest one is enough to be considered in the transient analysis of the induced voltage.

3.2.4 Effect of lead wire inductance It is wellknown that a transient response in a circuit is significantly affected by the impedance of a lead wire used for grounding, connecting circuits and measurements ${ }^{(2)(3)}$. In Ref. (2), 


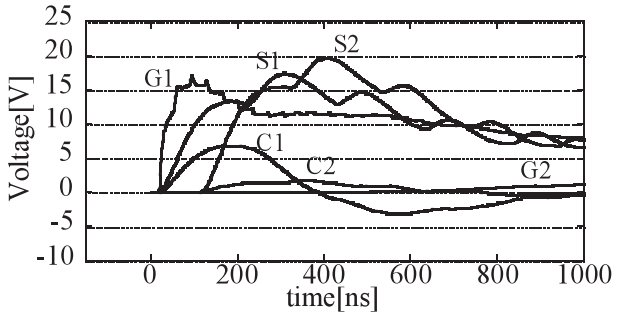

(1) No mutual coupling (Case C0-12)

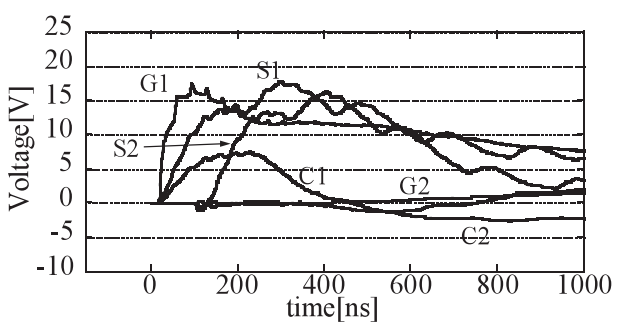

(2) Mutual coupling (Case C1-12)

(a) Sending-end grounding

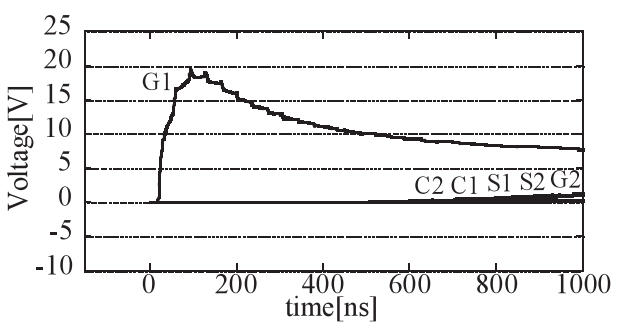

(1) No mutual coupling (Case C0-13)

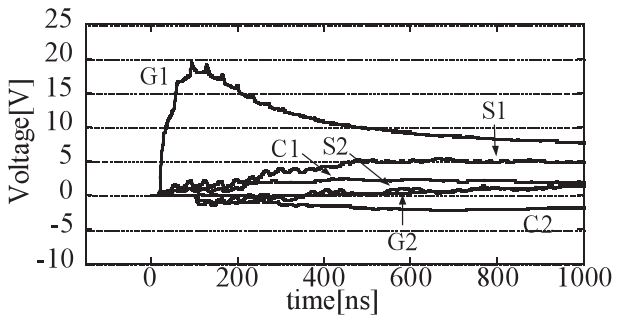

(2) Mutual coupling (Case C1-13)

(b) Receiving-end grounding

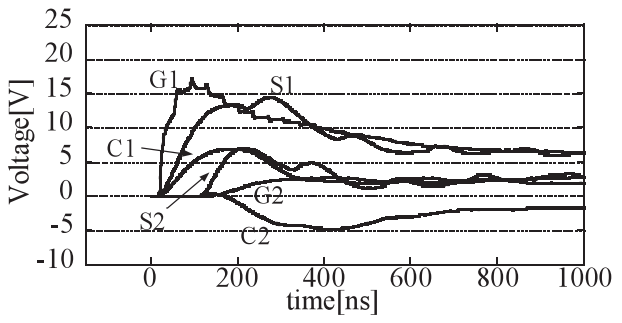

(1) No mutual coupling (Case C0-14)

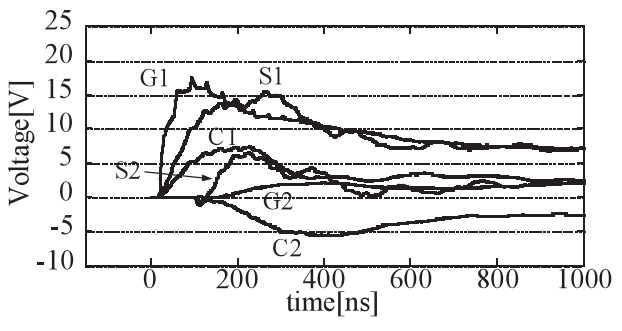

(2) Mutual coupling (Case C1-14)

(c) Both-end grounding

Fig. 11. Effect of lead wire inductance on cable voltages

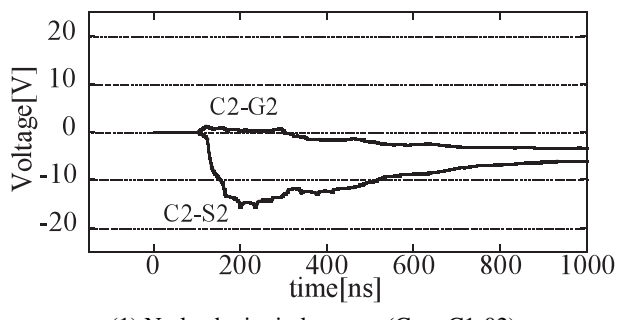

(1) No lead wire inductance (Case C1-02)

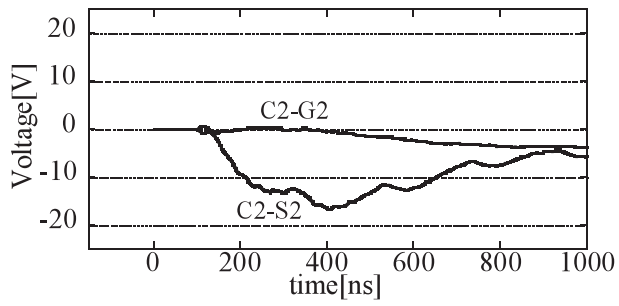

(2) Lead wire inductance considered (Case C1-12)

(a) Sending-end grounding

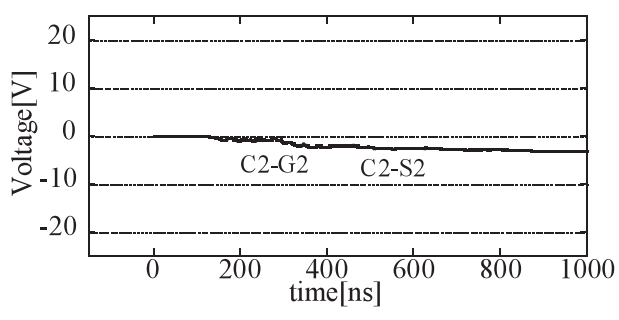

(1) No lead wire inductance (Case C1-03)

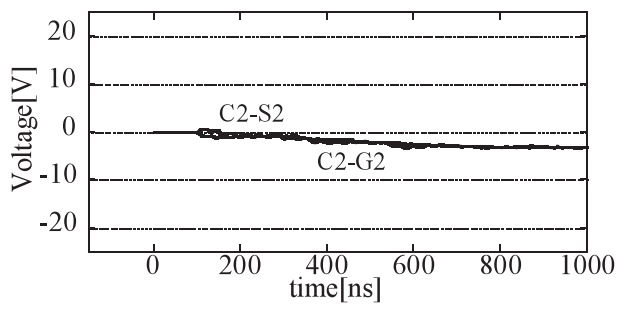

(2) Lead wire inductance considered (Case C1-13) (b) Receiving-end grounding

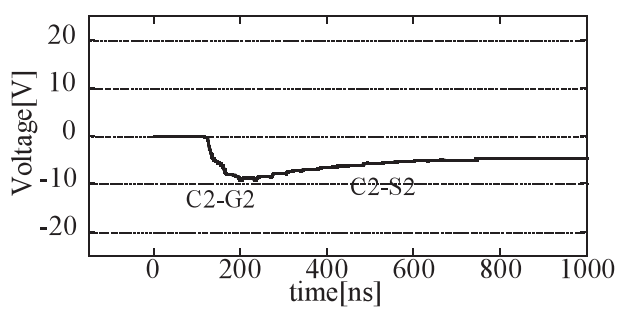

(1) No lead wire inductance (Case C1-04)

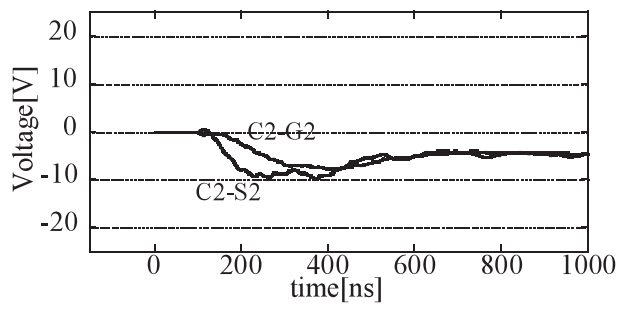

(2) Lead wire inductance considered (Case C1-14) (c) Both-end grounding

Fig. 12. Core voltages to sheath and counterpoise 
it is said that the grounding of the metalic sheath of a control cable may not be effective at all during a high frequency transient because of the grounding lead inductance.

Figure 11 shows the effect of the grounding lead inductance assuming $1 \mu \mathrm{H} / \mathrm{m}$ on voltages and currents of a control cable. It is observed in Table 1 that the inductance of $5 \mu \mathrm{H}$, corresponding to the $5 \mathrm{~m}$ grounding lead, reduces a current flowing into the sheath and the core voltages nearly a half for the case of no mutual coupling. However Fig. 11 with mutual coupling shows that the cable voltages are not much different from those in Fig. 8. The reason for this is readily explained that the high impedance of the grounding lead due to the inductance decreases the current flowing into the sheath and thus the current on the counterpoise increases which induces more voltages on the cable core. Similarly to Sect. 3.2.1. the largest core voltage at the receiving end is observed when the sheath both ends are grounded. Thus, it is concluded that the receiving end grounding is prefered even when a lead wire inductance is considered.

The above observations agree with those explained in Ref. (2) that sheath grounding becomes not effective for a transient due to the inductance of a lead wire.

3.2.5 Core voltages to sheath and counterpoise The previous sections concern voltages to the zero potential surface to investigate the effect of sheath grounding. In practice, a voltage difference from the core of a control cable to the sheath or to a counterpoise is used as the core voltage. Fig. 12 show the voltage difference. It is clear that the voltage difference from the core to the sheath is nearly the same as that to the counterpoise independently from the lead wire inductance. However, the voltage difference to the sheath is entirely dependent on the sheath grounding, and is greater than that to the counterpoise. The voltage difference is the greatest in the case of the sheath sending-end grounding. The receiving-end grounding shows the smallest difference. The results again suggest that the receiving-end grounding is better than the both-end grounding.

\section{Conclusinons}

Based on investigations of lightning surges on a control cable incoming through a grounding mesh, the following remarks have been obtained.

(1) So-called induced voltages on a control cable is a sum of an induced voltage due to a current on a counterpoise and of a voltage transfered from the counterpoise. When the both ends of the cable are grounded, a circulating current in a closed loop composed of the cable sheath and the counterpoise affects significantly the core voltage at the receiving end which becomes the largest among various sheath grounding.

(2) When the parallel length of the control cable and the counterpoise is long, current decrease along the counterpoise should be taken into account to evaluate an induced voltage to the cable core due to mutual coupling between the cable and the counterpoise. Otherwise, an excessive induced voltage due to the mutual coupling is expected.

(3) A lightning current incoming into a node of a grounding mesh distributes to every branches (counterpoises) connected at the node, and thus currents flowing into the cable sheath and the counterpoise parallel to the cable become smaller. This results in smaller voltages on the cable. Thus, an analysis assuming a single counterpoise gives the severest voltage on the cable, that is on a safer side

(4) The inductance of a grounding lead reduces a current flowing into the sheath because of its high impedance for a high frequency transient. At the same time, a current flowing into the counterpoise parallel to the cable increases and the current results in a higher induced voltage to the cable core due to mutual coupling between the cable and the counterpoise.

(Manuscript received Feb. 27, 2006, revised July 3, 2006)

\section{References}

( 1 ) T. Hasegawa, Y. Tokko, Y. Kanamori, I. Ii, and S. Sekioka: "A fact finding analysis of lightning interference on low-voltage control circuits in generator stations and substations", IEEJ 1990 Annual Conference, Paper 1228 (19903) (in Japanese)

(2) "Technologies of countermeasures against surges on protection relays and control systems", ERTA Report, Vol.57, No.3 (2002-1) (in Japanese)

( 3 ) CIGRE WG 36. 04: Guide on EMC in Power Plants and Substations, CIGRE Pub. 124 (1997)

(4) IEC: Electromagnetic Compatibility Part 6-5 Generic Std. Immunity for power station and substation enviroments IEC TC 61000-6-5 (2001)

( 5 ) Japanese Electotech. Committee: "Standard of Test Voltages for LowVoltage Control Circuits in Power Stations and Substations", JEC-01032005, Denki-Shoin Pub. Co., Tokyo, Japan (2005-11) (in Japanese)

(6) A. Ametani, M. Nayle, S. Sekioka, and T. Sonoda: "Basic investigation of wave propagation characteristics of an underground bare conductor", ICEE 2002 Proceedings, pp.2141-2146, Jeju, Korea (2002-7)

( 7 ) A. Ametani, T. Okumura, N. Nagaoka, and N. Mori: "Experimental investigation of a transient induced voltage to an overhead control cable from a grounding circuit”, CIRED2003, Paper No.2.4, Barcelone, Spain (2003-5)

( 8 ) T. Sonoda, Y. Takeuchi, S. Sekioka, N. Nagaoka, and A. Ametani: "Induced surge characteristics from a counterpoise to an overhead circuit", IEEJ Trans. $P E$, Vol.123, No.11, pp.1340-1349 (2003-11)

( 9 ) H. Motoyama: "Lightning surege characteristics of low voltage and control circuits (I)", CRIEPI Report H04011 (2005-5) (in Japanese)

(10) D. Soyama, Y. Ishibashi, N. Nagaoka, and A. Ametani: "Modeling of a buried conductor for an electromagnetic trasient simulation", ICEE 2005, Paper SM1-04, Kunming, China (2005-7)

(11) H. Isogai, A. Ametani, and Y. Hosokawa: "An investigation of induced voltages to an underground gas pipeline from an overhead transmission line", IEEJ Trans. PE, Vol.126, No.1, pp.43-50 (2006-1) (in Japanese)

(12) A. Ametani: Cable Parameters Rule Book, Bonneville Power Administration, Portland, USA (1994-4)

(13) A. Ametani: Distributed-Parameter Circuit Theory, Corona Pub. Co., Tokyo (1990) (in Japanese)

Akihiro Ametani (Member) was born on February 14, 1994. He re-

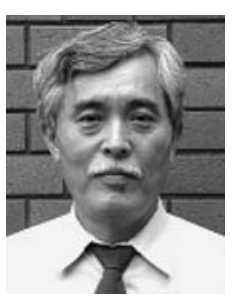
cevived Ph.D. degree from UMIST, Manchester in 1973. He was with the UMIST from 1971 to 1974 , and Bonneville Power Administration to develop EMTP for summers from 1976 to 1981 . He has been a professor at Doshisha University since 1985 and was a professor at the Chatholic University of Leaven, Belgium in 1988. He was the Director of the Institute of Science and Engineering from 1996 to 1998, and Dean of Library and Computer/Information Center in Doshisha University from 1998 to 2001. Dr. Ametani is a Chartered Engineer in U.K., a Fellow of IEE and IEEE. 
Norikazu Taki (Student Member) was born in Aichi, Japan on Jan-

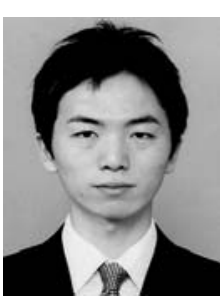
uary 6, 1982. He received B.Eng. and M.Eng. degrees from Doshisha University in 2004 and 2006 respectively. He is employed by RICOH since April, 2006.

Daisuke Miyazaki (Student Member) was born in Osaka, Japan on

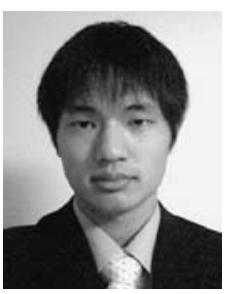
Doshisha University in 2006. Now he is an M.Eng. student in the Graduate School of the same university.
Naoto Nagaoka (Member) was born in Nagoya, Japan on October

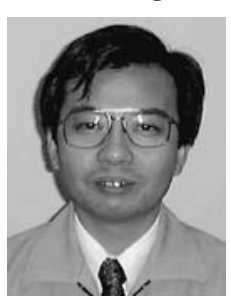
21, 1975. He received B.S., M.S. and Dr.Eng. degrees all from Doshisha University in 1980, 1982 and 1993 respectively. He joined the Doshisha University in 1985, and has been a professor since 1999. Dr. Nagaoka is a member of IEE and IEEE.

Shigemitu Okabe (Member) received Ph.D. degrees from Tokyo Uni-

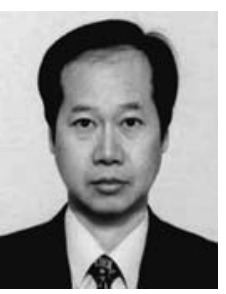
versity. Graduate School in Marc 1986. Since April 1986, he is with Tokyo Electric Power Co. In 1992, he was a research fellow at The Technical University of Munchen. Presently he is a Manager of the high voltage and insulation technology group of the $\mathrm{R} \&$ D Center of Tokyo Electric Power Co. Dr. Okabe is a member of the IEEE. 